\title{
Perfil Epidemiológico e Análise de Sobrevida em Pacientes com Neoplasia de Pulmão tratados em um Hospital Público no Município de São Bernardo do Campo - SP: um Estudo Retrospectivo
}

\author{
Epidemiological Profile and Survival Analysis in Patients with Lung Neoplasia treated at a Public \\ Hospital in the Municipality of São Bernardo do Campo - SP: a Retrospective Study \\ Stéphane Tomás da $\mathrm{Luz}^{1}$, Patricia Xavier Santi ${ }^{2,3}$, Daniel de Iracema Gomes Cubero ${ }^{3,4}$, Jean Henri Maselli Schoueri ${ }^{5}$, Auro del Giglio ${ }^{3,6}$, \\ Claudia Vaz de Melo Sette ${ }^{3,4}$
}

\begin{abstract}
Com citar: Luz ST, Santi PX, Cubero DIG, Schoueri JHM, Giglio A, Sette CVM. Perfil Epidemiológico e Análise de Sobrevida em Pacientes com Neoplasia de Pulmão tratados em um Hospital Público no Município de São Bernardo do Campo - SP: um Estudo Retrospectivo. Clin Onc Let. 2020; Ahead of Print. http://dx.doi.org/10.4322/col.2019.004
\end{abstract}

\begin{abstract}
RESUMO
Introdução: O câncer de pulmão (CP) é uma das neoplasias mais comuns de todos os tumores malignos, com letalidade e incidência crescente principalmente entre mulheres. Objetivos: Avaliar fatores epidemiológicos e sobrevida de pacientes com CP de um hospital público de São Bernardo do Campo-SP-Brasil e correlacioná-los com dados apresentados em estudos na área. Método: Estudo retrospectivo a partir da análise dos prontuários de pacientes diagnosticados com CP (CID 10: C34), acompanhados no Hospital Anchieta de São Bernardo do Campo - SP, no período de 01/06/2016 a 01/07/18. Para análise de sobrevida, utilizou-se as curvas de Kaplan-Meier com teste de log rank. O software utilizado foi o Stata ${ }^{\circledR}$ versão 11.0 Resultados: Dos 80 pacientes incluídos, a média de idade foi de 68 anos entre homens e 64 entre mulheres, sendo $41 \%$ do tipo histológico adenocarcinoma e 34\% de CEC. Cerca de $76 \%$ eram avançadas ao diagnóstico e quase 41\% com ECOG 1 ao diagnóstico. Quanto à quimioterapia, 35\% receberam primeira linha baseada em platina no adenocarcinoma, $53 \%$ no CEC e $56 \%$ no pequenas células. Na amostra estudada, $56 \%$ evoluíram a óbito, sendo que $18 \%$ após o diagnóstico, sem ter recebido qualquer linha de tratamento. A mediana de sobrevida global foi 11 meses. Conclusão: Os pacientes atendidos neste hospital pertencente ao sistema público brasileiro apresentaram sobrevida muito pequena, divergindo dos resultados encontrados na literatura, provavelmente devido à dificuldade de acesso ao sistema de saúde especializado e do estágio avançado do diagnóstico.
\end{abstract}

Palavras chaves: Câncer de Pulmão; Histologia; Quimioterapia; Sobrevida

\begin{abstract}
Introduction: Lung cancer is the most common malignant neoplasm in the world, with lethality and increasing incidence mainly among women. Objectives: To evaluate epidemiological factors and survival of patients with lung cancer in a public hospital in São Bernardo do Campo-SP-Brazil and to correlate them with the data presented in studies in the area. Methods: Retrospective study based on the analysis of medical records of patients diagnosed with CID 10: C34 lung cancer, followed at Anchieta Hospital in São Bernardo do Campo, SP, Brazil, from 06/01/2016 to 01 / 07/18. For survival analysis, Kaplan-Meier curves were used with log rank test and the software used was Stata ${ }^{\circledR}$ version 11.0. Results: The study included 80 patients with lung cancer with mean age of 68 years among men and 64 among women, with $41 \%$ of histological type adenocarcinoma and $34 \%$ of CPB. About $76 \%$ were already advanced at diagnosis and almost $41 \%$ with
\end{abstract}

\footnotetext{
${ }^{1}$ Médica Oncologista do Hospital Anchieta - São Bernardo do Campo, São Paulo, Brasil

${ }^{2}$ Chefe do Departamento de Oncologia do Hospital Estadual Mário Covas - Santo André, São Paulo, Brasil

${ }^{3}$ Centro de Estudo e Pesquisa de Hematologia e Oncologia, Faculdade de Medicina do ABC - Santo André, São Paulo, Brasi

${ }^{4}$ Professor assistente da disciplina de Oncologia da Faculdade de Medicina do ABC - Santo André, São Paulo, Brasil

${ }^{5}$ Acadêmico - Santo André, São Paulo, Brasil

${ }^{6}$ Professor titular de Hematologia e Oncologia da Faculdade de Medicina do ABC - Santo André, São Paulo, Brasil

Financiamento: Não houve financiamento para o presente estudo.

Conflito de interesse: Os autores declararam não haver conflitos de interesse que precisam ser informados.
} 
ECOG 1 at diagnosis. Regarding chemotherapy, 35\% received platinum-based first line in adenocarcinoma, 53\% in CPB and $56 \%$ in small cells. In the sample studied, $56 \%$ died, $18 \%$ after the diagnosis, without having received any treatment line. In the study period, the median overall survival was 11 months. Conclusion: The patients treated at this hospital belonging to the Brazilian public system had a very short survival, diverging from the results found in the literature, probably due to the difficulty of accessing the specialized health system and the advanced stage of diagnosis.

Key words: Lung Cancer; Histology; Chemotherapy; Survival

\section{INTRODUÇÃO}

O câncer de pulmão é uma das neoplasias mais comuns de todos os tumores malignos, com letalidade e incidência crescente principalmente entre as mulheres. ${ }^{1}$ Corresponde a $13,5 \%$ de todos os tipos de câncer, ficando atrás do de mama e próstata. A estimativa mundial em 2015 foi de 2,019 milhões de novos casos, distribuídos em 1,38 milhão entre homens e 640 mil casos entre mulheres. ${ }^{2}$

Estima-se que no Brasil, segundo dados do Ministério da Saúde, que no ano de 2018 apontam para 31.270 novos casos diagnosticados, sendo o segundo mais comum entre homens e o quarto em mulheres, com taxa de incidência de 18,16 casos para 100.000 habitantes/ano nos homens e $11,81 / 100.000$ entre as mulheres. ${ }^{3,4}$

Apesar de mais frequente no sexo masculino, sua incidência no homem se estabilizou ou diminuiu, enquanto nas mulheres vem apresentando um aumento importante nas últimas décadas. Na maioria das vezes o diagnóstico é tardio, quando a doença já se encontra em fases avançadas, impedindo assim, o tratamento curativo. Somente $20 \%$ dos casos são diagnosticados em fases iniciais. ${ }^{2,5}$

O câncer de pulmão além de ser frequente, é altamente letal. Estimativas de 2015, publicadas pelo Global Burden of Disease, apontam cerca de 1,7 milhão de óbitos devido neoplasias pulmonares a cada ano, sendo 1,2 milhão entre os homens e 517 mil entre as mulheres, com uma taxa de incidência padronizada de 26,6 mortes por 100.000 habitantes/ano. ${ }^{6}$ No Brasil, ocorreram em 2015, 15.514 óbitos por câncer de pulmão em homens e 10.978 em mulheres. ${ }^{3}$

Altamente letal, a sobrevida média cumulativa total em cinco anos varia de 13 e $21 \%$ em países desenvolvidos e 7 e $10 \%$ nos países em desenvolvimento. ${ }^{2}$

O tabagismo é principal fator de risco para desenvolver câncer de pulmão. ${ }^{1} \mathrm{O}$ câncer de pulmão aumenta na proporção direta do número de cigarros que se fuma por dia, entretanto, estudos epidemiológicos apontam que cerca de um terço dos casos de câncer de pulmão em não fumantes estava associado ao tabagismo passivo. A exposição à poluição do ar, infecções pulmonares de repetição, suplementação de altas doses de vitamina $A$, doença obstrutiva crônica, fatores genéticos e história familiar de câncer de pulmão, favorecem para o desenvolvimento desse tipo de câncer..$^{5,7}$ Outros fatores incluem a exposição ao amianto, a certos metais como cromo, cadmio, arsênio, alguns produtos químicos orgânicos, radiação, poluição do ar e escape de diesel. ${ }^{5,7}$ A susceptibilidade genética desempenha papel no desenvolvimento do câncer de pulmão, principalmente para os de início precoce. ${ }^{8}$

O câncer de pulmão é classificado em dois grupos: carcinoma de pequenas células e os carcinoma não pequenas células, sendo que este último, compreende o carcinoma de células escamosas ou carcinoma espinocelular, adenocarcinoma e carcinoma indiferenciado de grandes células (neuroendócrino e não neuroendócrino), carcinoma adenoescamoso, carcinoma pleomórfico e sarcomatóide, e tumor carcinoide. ${ }^{9}$ Nos últimos anos, o diagnóstico tem sido feito cada vez mais com base molecular, evidenciando estudos específicos as diferentes famílias de genes e o evento mutagênico envolvido, assim caracterizando cada tipo histológico com suas alterações moleculares especificas..$^{9,10}$

O estadiamento do câncer de pulmão pelo sistema TNM vem sofrendo modificações desde 1070. Atualmente é realizado baseando-se na extensão anatômica da neoplasia, representada e detalhada pelo sistema TNM, 8 a edição, onde o T refere-se ao tamanho do tumor, o $\mathrm{N}$ ao número e características dos linfonodos acometidos pela doença e $\mathrm{M}$ ao número e localização das metástases tumorais. ${ }^{11}$

O tratamento do câncer de pulmão, quando se trata de doença localizada, sem disseminação para fora do pulmão, é cirúrgico. ${ }^{9,10}$ Tumores restritos ao pulmão, nos estadios I e II, apresentam chances de curas com sobrevida de cinco anos variando de 50 a $80 \%$, segundo relatos na literatura mundial, e o tratamento cirúrgico é ainda considerado a terapia de escolha. ${ }^{9,12}$ Alguns estudos realizados pelo Lung Cancer Study Group, mostraram que a quimioterapia adjuvante (pós operatória) em esquemas baseados em cisplatina, apresentou aumento de sobrevida 
livre de recidiva (6 a 7 meses), entretanto, a sobrevida global não foi alterada. ${ }^{1}$ Os pacientes com estadio III (doença avançada locorregional) devem ter avaliação multidisciplinar para determinar tratamento, incluindo avaliação da equipe de cirurgia torácica e radioterapia. Os pacientes com estadio IV (doença metastática), devem sem avaliados individualmente para definição de tratamento sistêmico paliativo. ${ }^{1}$

A análise do perfil epidemiológico na população com câncer de pulmão com seus respectivos fatores de risco, prognósticos e resultados de tratamento, são de fundamental importância para identificar quais estratégias podem ser adotadas a fim de reduzir as altas taxas de incidência desta neoplasia e aprimorar as opções terapêuticas.

Deste modo, o presente estudo tem como objetivo identificar o perfil epidemiológico dos pacientes portadores de câncer de pulmão, atendidos em um hospital de público no município de São Bernardo do Campo - São Paulo. Também serão apresentados resultados do tratamento instituído quanto à sobrevida livre de progressão e sobrevida global.

\section{PACIENTES E MÉTODOS}

Após aprovação do trabalho pelo Comitê de Ética e Pesquisa do Hospital Anchieta, foi realizado estudo retrospectivo a partir da análise dos prontuários de pacientes diagnosticados com neoplasia de pulmão (Código Internacional de Doenças 10a edição-CID 10: C34), acompanhados no Hospital Anchieta de São Bernardo do Campo - SP, no período de 01/06/2016 a 01/07/18.

Foram incluídos todos os pacientes maiores de 18 anos, diagnosticados com neoplasia de pulmão atendidos neste período. Os dados coletados foram tabulados, descritos através de médias, medianas, frequências absolutas e relativas. Para análise de sobrevida, utilizou-se as curvas de Kaplan-Meier com teste de log rank. O nível de significância assumido foi de $5 \%$ e o software utilizado para as análises foi o Stata ${ }^{\circledR}$ versão 11.0

\section{RESULTADOS}

Foi realizada uma análise retrospectiva dos pacientes portadores de Neoplasia de Pulmão tratados em um hospital público no município de São Bernardo do Campo - SP. O período de análise retrospectiva foi de 01/06/2016 a 01/07/2018. Foram recrutados 97 pacientes, porém 17 pacientes foram excluídos do estudo, pois não passaram em uma primeira consulta no serviço. Foram analisados 80 pacientes, dentro os quais 44 eram do sexo masculino, correspondendo a $55 \%$ da amostra e 36 eram do sexo feminino, correspondendo a $45 \%$. A idade média foi de 68 anos entre os homens e 64 entre as mulheres. 0 tabagismo foi evidenciado em $66 \%$ dos pacientes, sendo a prevalência de $62 \%$ entre os homens e $38 \%$ entre as mulheres (tabela 1 ).

Tabela 1. Características demográficas e clínicas

\begin{tabular}{|c|c|c|c|}
\hline Variável & Categorias & & \\
\hline \multirow[t]{3}{*}{ Sexo } & & $n$ & $\%$ \\
\hline & Masculino & 44 & $55 \%$ \\
\hline & Feminino & 36 & $45 \%$ \\
\hline \multirow[t]{3}{*}{ Idade } & & média & mediana \\
\hline & Masculino & 68 & 68 \\
\hline & Feminino & 64 & 62 \\
\hline \multirow[t]{6}{*}{ ECOG } & & n & $\%$ \\
\hline & 0 & 18 & $23 \%$ \\
\hline & 1 & 34 & $43 \%$ \\
\hline & 2 & 17 & $21 \%$ \\
\hline & 3 & 9 & $11 \%$ \\
\hline & 4 & 2 & $3 \%$ \\
\hline \multirow[t]{4}{*}{ Tabagismo } & & $\mathbf{n}$ & $\%$ \\
\hline & Sim & 53 & $66 \%$ \\
\hline & Não & 10 & $13 \%$ \\
\hline & Desconhecido & 17 & $21 \%$ \\
\hline
\end{tabular}


Tabela 1. Continuação...

\begin{tabular}{|c|c|c|c|}
\hline Variável & Categorias & & \\
\hline \multirow[t]{5}{*}{ Mutação } & & $\mathbf{n}$ & $\%$ \\
\hline & Sem Resultado & 61 & $76 \%$ \\
\hline & Inconclusivo & 5 & $6 \%$ \\
\hline & Negativo & 8 & $10 \%$ \\
\hline & Positivo & 6 & $8 \%$ \\
\hline \multirow[t]{4}{*}{ Óbito } & & $n$ & $\%$ \\
\hline & Sim & 45 & $56 \%$ \\
\hline & Não & 20 & $25 \%$ \\
\hline & Desconhecido & 15 & $19 \%$ \\
\hline
\end{tabular}

Os tipos histológicos mais comuns foram o Adenocarcinoma 41\%, seguido do Carcinoma Espinocelular (CEC) $34 \%$ (tabela 2). Sendo o Adenocarcinoma o mais prevalente entre as mulheres e o CEC entre os homens (tabela 3).

Tabela 2. Incidência por tipo histológico

\begin{tabular}{cccc}
\hline Tipo histológico & $\mathbf{n}$ & \% & $41 \%$ \\
\hline Adenocarcinoma & 33 & $34 \%$ & $15 \%$ \\
CEC & 27 & $1 \%$ & $9 \%$ \\
Pequenas células & 12 & 1 & \\
Misto & 1 & & \\
Outros & 7 & & \\
\hline
\end{tabular}

Tabela 3. Incidência histológica por Sexo

\begin{tabular}{|c|c|c|c|}
\hline Masculino & & $\mathbf{n}$ & $\%$ \\
\hline & Adenocarcinoma & 14 & $35 \%$ \\
\hline & CEC & 19 & $48 \%$ \\
\hline & Pequenas células & 7 & $18 \%$ \\
\hline \multirow[t]{4}{*}{ Feminino } & & $\mathbf{n}$ & $\%$ \\
\hline & Adenocarcinoma & 19 & $58 \%$ \\
\hline & CEC & 9 & $27 \%$ \\
\hline & Pequenas células & 5 & $15 \%$ \\
\hline
\end{tabular}

Dos 80 pacientes estudados, 66 (83\%) realizaram algum tipo de tratamento para a neoplasia. Sendo que 73 (91\%) apresentavam, ao diagnóstico, estadio III e IV, e os outros 7 (9\%) apresentavam estadio I e II (tabela 4).

Tabela 4. Incidência por estadio clínico

\begin{tabular}{cccc}
\hline Estadio & $\mathbf{n}$ & $\%$ & $3 \%$ \\
\hline I & 2 & $6 \%$ & \\
II & 5 & $15 \%$ & $76 \%$ \\
III & 12 & 61 & \\
IV & 61 & & \\
\hline
\end{tabular}

Da amostra estudada, 14 pacientes não receberam tratamento, 8 (57\%) apresentavam performance status ruim, os $6(43 \%)$ restantes, entraram em seguimento desde a primeira consulta, pois receberam tratamento cirúrgico exclusivo ou decidiram tratar em outro serviço.

A respeito da pesquisa de mutação genética (EGFR, ALK, ROSS, BRAF, KRAS e NRAS) e $P D L 1$, dentre os pacientes com diagnóstico histológico de CEC, $96 \%$ não apresentavam resultado ou pedido de mutação descrita em prontuário e $4 \%$ apresentavam resultado negativo para mutação. Dos pacientes portadores de Adenocarcinoma, $52 \%$ não 
possuíam resultado ou solicitação de pesquisa em prontuário, 5 (15\%) apresentavam resultado inconclusivo, 6 (18\%) apresentavam resultado negativo e $5(15 \%)$ apresentavam resultado positivo para alguma mutação genética, dentre as quais 3 (60\%) apresentavam mutação do EGFR, 1 (20\%) do ALK e 1 (20\%) mutação do BRAF.

Em relação ao tratamento, 50 pacientes com estadio clínico IV (75\%) foram submetidos à quimioterapia exclusiva, onde os esquemas mais utilizados, de acordo com o tipo histológico, estão descritos na tabela 5. Sobre os demais (16 pacientes), 4 (6\%) realizaram quimioterapia associada à radioterapia, $3(5 \%)$ foram submetidos à cirurgia associada a quimioterapia neoadjuvante/adjuvante, 4 (6\%) foram encaminhados para estudo clinico, 3 (5\%) receberam tratamento com radioterapia paliativa e $2(3 \%)$ receberam radioterapia exclusiva.

Tabela 5. Tratamentos por tipo histológico

\begin{tabular}{cccc}
\hline & Adenocarcinoma & CEC & Pequenas Células \\
\hline 1ㅇ linha de quimioterapia & Carboplatina + Taxol (35\%) & Carboplatina + Gemcitabina (53\%) & Cisplatina + Etoposideo (56\%) \\
& Sem Tratamento (65\%) & Sem Tratamento (41\%) & Sem Tratamento (44\%) \\
20 linha de quimioterapia & Docetaxel (30\%) & Docetaxel (24\%) & Sem Tratamento (67\%) \\
3 linha de quimioterapia & Sem Tratamento (60\%) & Sem Tratamento (41\%) & .
\end{tabular}

Dentre os 66 pacientes tratados, 51 (77\%) apresentavam metástases no momento do diagnóstico, vários com múltiplos órgãos afetados. Os principais sítios foram: pulmão, cérebro, osso, adrenal e fígado, nessa ordem.

Dos pacientes estudados, 18 (23\%) apresentavam Eastern Cooperative Oncology Group Performance Status (ECOG) igual a 0, 34 (43\%) um ECOG igual a 1, 17 (21\%) um ECOG igual a 2, e 11 (14\%) um ECOG entre 3 e 4. A sobrevida livre de progressão mediana, nos pacientes estadio IV, foi de 6 meses no nosso estudo (gráfico 1).

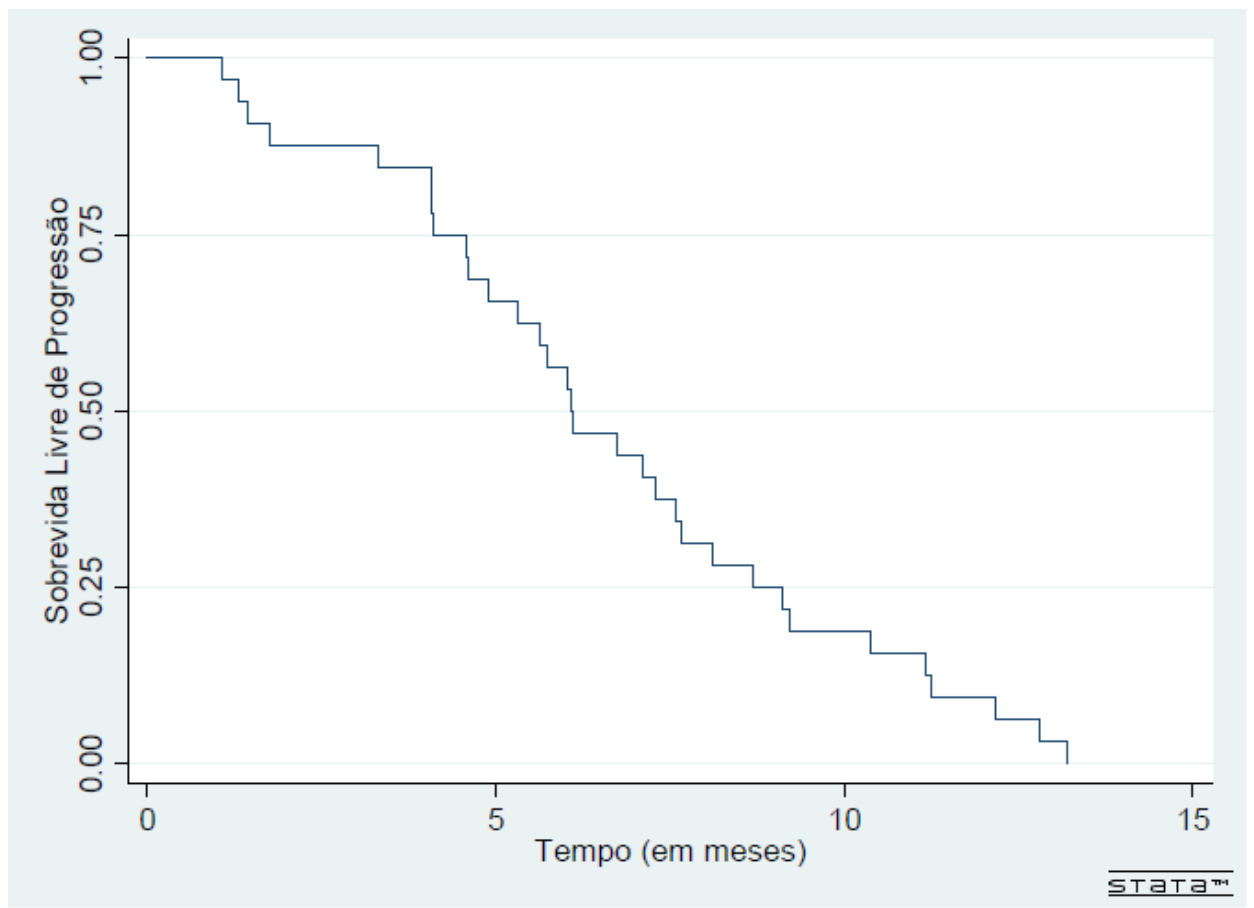

Gráfico 1. Sobrevida livre de progressão

Em relação a sobrevida global nos pacientes estadio IV, a mediana do nosso estudo foi de cerca de 11 meses (gráfico 2). Ainda em relação a sobrevida global, podemos observar os dados de acordo com o tipo histológico, mostrando uma sobrevida global maior naqueles pacientes com adenocarcinoma (gráfico 3). Comparando-se as curvas, podemos observar uma diferença estatisticamente significativa entre os tipos histológicos $(p=0,03)$. 


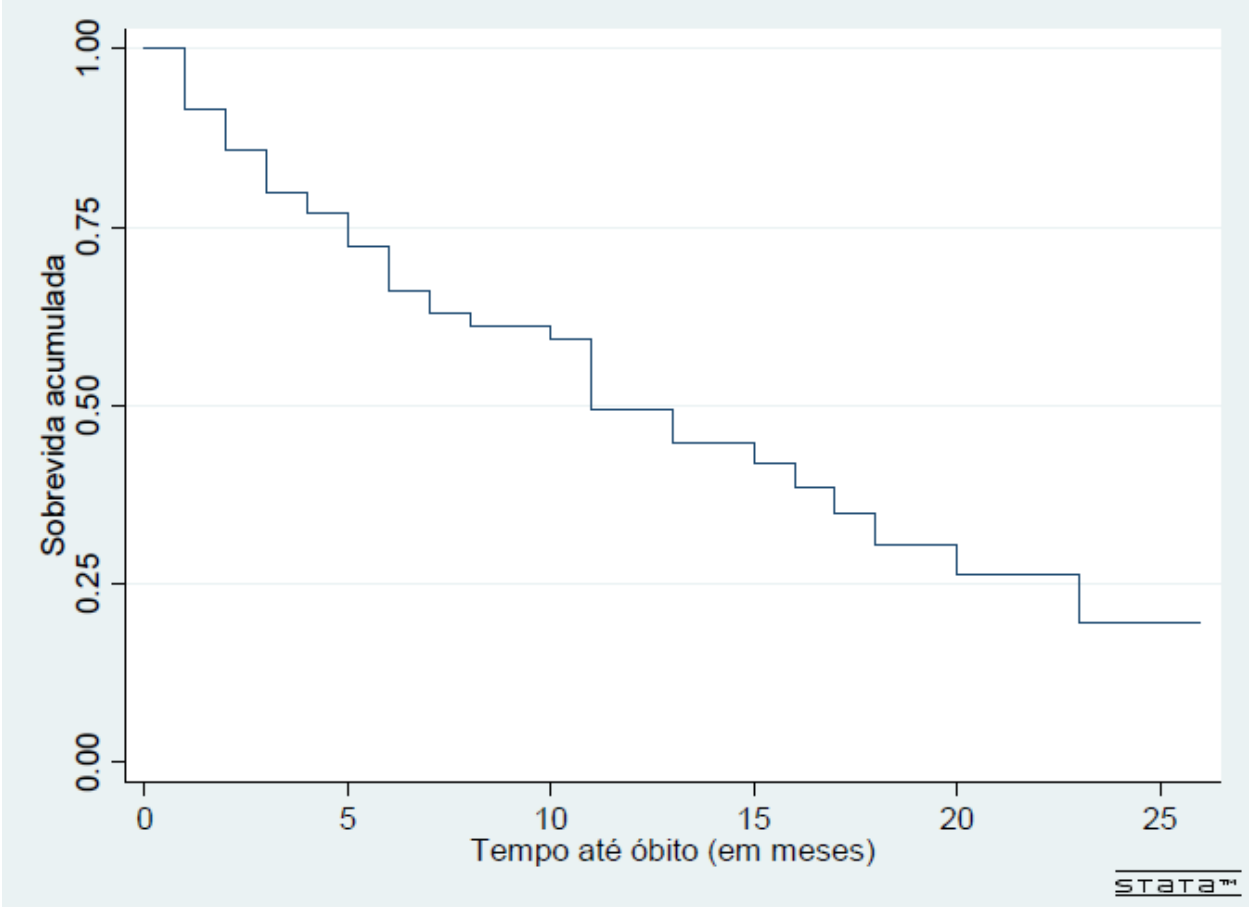

Gráfico 2. Sobrevida Global

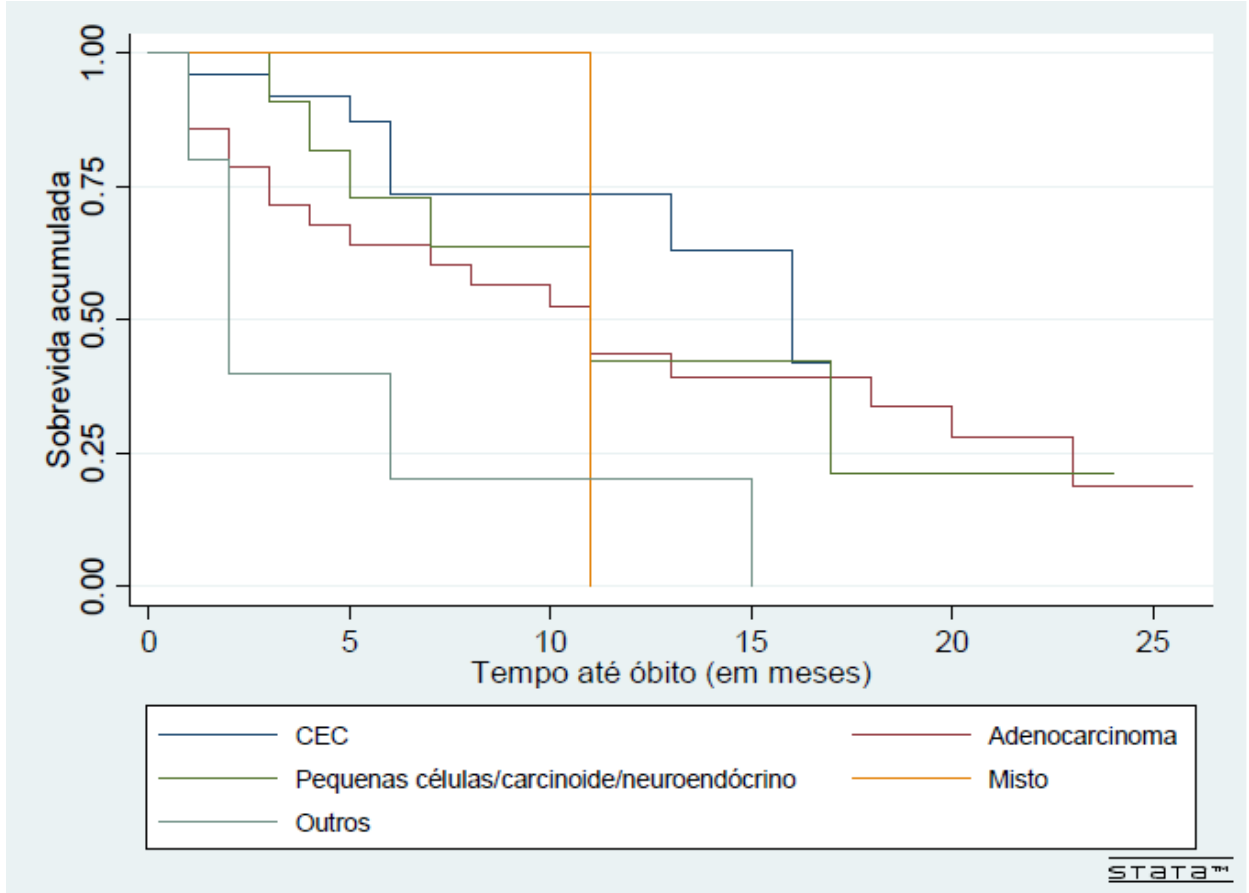

Gráfico 3. Sobrevida Global por tipo histológico $(p 0,03)$

Até a finalização da análise dos dados do estudo, 45 pacientes (56\%) foram a óbito, sendo destes, 20 (44\%) após a primeira linha de tratamento, $13(29 \%)$ após a segunda linha, $4(9 \%)$ após a terceira ou mais linha de tratamento e $8(18 \%)$ após o diagnóstico sem terem recebido qualquer esquema de tratamento.

Encontram-se em seguimento devido doença estável, término de adjuvância ou férias de quimioterapia, 14 pacientes (18\%). Dos pacientes diagnosticados com neoplasia de pulmão no período abordado pelo estudo, 6 (8\%) 
encontram-se atualmente em tratamento quimioterápico. Optaram por tratar em outro serviço 4 (5\%) pacientes e 11 pacientes (14\%), perderam o seguimento no serviço e não conseguimos contato telefônico para maiores informações.

\section{DISCUSSÃO}

O câncer de pulmão é uma das neoplasias com um dos menores índices de sobrevida, com variabilidade conforme a população em estudo. É a neoplasia maligna mais incidente em todo o mundo, com letalidade e incidência crescente principalmente entre as mulheres, concordando com a literatura. ${ }^{13}$

Quanto ao tipo histológico, notamos uma baixa incidência do carcinoma de pequenas células, conforme tem mostrado a literatura. A distribuição segundo Ismael et. al. e Westphal et. al., tem ficado entre $25,5 \%$ e $62,8 \%$ para o carcinoma espinocelular, entre $24,7 \%$ e $27,4 \%$ para o adenocarcinoma e $9,1 \%$ e $10,5 \%$ para o carcinoma de pequenas células. ${ }^{13,14}$

Nosso estudo nesses 2 anos manteve o adenocarcinoma em primeiro lugar, mas com valores muito próximos ao CEC, conforme Novaes et.al. Apesar de a agressividade do tumor variar com o tipo histológico, não observamos diferença significante na sobrevida, entre os vários tipos. Em contrapartida, a alta prevalência de estádios avançados ao diagnóstico reflete a sintomatologia e diagnóstico tardio e a alta agressividade de determinados tipos histológicos. Além disso, explica o número reduzido de pacientes submetidos ao tratamento cirúrgico, indicação incontestável nos estadios iniciais e reservada nos estádios mais avançados. ${ }^{9,13,14} \mathrm{Na}$ literatura, somente cerca de $20 \%$ dos casos têm critérios de operabilidade ao diagnóstico, mesmo nos países de primeiro mundo. ${ }^{10} \mathrm{Em}$ nosso estudo, a principal causa de não tratamento foi a performance status ruim. Da amostra total do nosso estudo, 33\% apresentavam performance status $2-3$ na escala do Eastern Cooperative Oncology Group (ECOG); corroborando com o estudo de Naime et al., que mostrou em um estudo com 562 pacientes, $47 \%$ apresentavam status 2-3 na escala do ECOG. ${ }^{15}$

Segundo Younes et al., a maioria dos pacientes foram tratados com quimioterapia de primeira linha e a segunda e terceira linha foi ofertada para a minoria. ${ }^{16}$ No nosso estudo, dos 66 pacientes tratados, 50 receberam quimioterapia exclusiva com tratamento em primeira linha com regime baseado em platina, conforme Younes et.al., porém, desses pacientes, apenas $24(48 \%)$ chegaram a receber a segunda ou mais linhas.

A curva de sobrevida global obtida reflete a alta agressividade do câncer de pulmão, demonstrando sobrevida média estimada em dois anos de apenas 11 meses. Em Westphal et al., a sobrevida estimada em 3 anos, foi de 11,7 meses. Estes dados reforçam a importância do diagnóstico precoce do câncer de pulmão, que propicia melhor prognóstico, com razoável taxa de cura.

O estudo em questão foi realizado em um único centro oncológico público, com casuística pequena. Dados mais abrangentes, em âmbito nacional, envolvendo múltiplos centros de referência em oncologia poderiam fornecer informações mais precisas para melhor correlação com a literatura internacional.

\section{CONCLUSÃO}

Sabe-se que estudos retrospectivos baseado em análise de prontuário têm suas limitações, como a falta de informações e o preenchimento incompleto do prontuário.

Como conclusões, observou-se que, em nosso meio, a incidência do câncer de pulmão continua sendo maior no homem, porém com aumento da incidência em mulheres. O tipo histológico predominante é o adenocarcinoma e o de menor incidência é o carcinoma de pequenas células. A maioria dos casos apresentam-se em estadio avançado ao diagnóstico. Os estadios iniciais que seriam candidatos a cirurgia, correspondem à menor número de pacientes, e a maioria dos pacientes necessitam de tratamento quimioterápico. A sobrevida global é baixa, porém quando comparamos o tipo histológico observamos uma maior sobrevida daqueles pacientes com adenocarcinoma.

Este estudo deve servir como ponto de partida para uma melhor avaliação das causas deste problema, abordando questões como diagnóstico precoce, acesso e tempo até os tratamentos preconizados. 


\section{BIBLIOGRAFIA}

1. Younes RN. Câncer de pulmão: atualização 2018. São Paulo: M\&ATD; 2018.

2. INCA: Instituto Nacional de Câncer [Internet]. Rio de Janeiro: INCA; 2017 [citado 2013 set 25]. Disponível em: http://www.inca.gov.br/estimativa/2018/estimativa-2018.pdf

3. Brasil. Ministério da Saúde. Departamento de informática do SUS. Estimativas da incidência e mortalidade por câncer no Brasil [Internet]. Brasília; 2014 [citado 2013 set 25]. Disponível em: http://www.datasus.gov.br

4. Brasil. Ministério da Saúde. Departamento de Informática do SUS. Sistema de Informações sobre mortalidade [Internet]. Brasília; 2017 [citado 2013 set 25]. Disponível em: http://www.datasus.gov.br

5. Alberg AJ, Brock MV, Samet JM. Epidemiology of lung cancer: looking to the future. J Clin Oncol. 2005;23(14):3175-85. http://dx.doi.org/10.1200/JCO.2005.10.462. PMid:15886304.

6. Global Burden of Disease Cancer Collaboration. Global, regional, and national cancer incidence, mortality, years of life lost, years lived with disability, and desability-adjusted life-years for 32 cancer groups, 1990 to 2015: a systematic analysis for the Global Burden of Disease study. JAMA Oncol. 2017;3(4):524-48. http://dx.doi.org/10.1001/jamaoncol.2016.5688. PMid:27918777.

7. Alberg AJ, Samet JM. Epidemiology of lung cancer. Chest. 2003;123(1, Suppl Suppl):21S-49S. http://dx.doi.org/10.1378/chest.123.1_suppl.21S. PMid:12527563.

8. Blot WJ, McLaughlin JK. Are women more susceptible to lung cancer? J Natl Cancer Inst. 2004;96(11):812-3. http://dx.doi.org/10.1093/jnci/djh180. PMid:15173256.

9. Novaes FT, Cataneo DC, Ruiz RL Jr, Defaveri J, Michelin OC, Cataneo AJM. Câncer de pulmão: histologia, estadio, tratamento e sobrevida. J Bras Pneumol. 2008;34(8):595-600. http://dx.doi.org/10.1590/S1806-37132008000800009.

10. Araujo LH, Baldotto C, Castro G Jr, et al. Câncer de pulmão no Brasil. J Bras Pneumol. 2018;44(1):55-64. http://dx.doi.org/10.1590/s1806-37562017000000135. PMid:29538545.

11. NCCN: National Comprehensive Cancer Network. TNM classification of malignant tumours [Internet]. 8th ed. Plymouth Meeting: NCCN; 2013 [citado 2013 set 25]. Disponível em: www.uicc.org.

12. NCCN: National Comprehensive Cancer Network. Clinical practice guidelines in oncology [Internet]. Plymouth Meeting: NCCN; 2008. vol. 1. Non-small cell lung cancer [citado 2013 set 25]. Disponível em: http://www.nccn.org/ professionals/physician_gls/PDF/nscl.pdf

13. Westphal FL, Lima LC, Andrade EO, Lima JC No, Silva AS, Carvalho BC. Caracteristicas de pacientes com câncer de pulmão na cidade de Manaus. J Bras Pneumol. 2009;35(2):157-63. http://dx.doi.org/10.1590/S1806-37132009000200009. PMid:19287919.

14. Isamael GFV, Coradazzi AL, Moraes FA No, et al. Aspectos clínicos e histopatológicos em câncer de pulmão: análise dos dados de uma instituição no interior paulistano entre 1997 e 2008. Revista Brasileira de Oncologia Clínica. 2010;7(22):72-8.

15. Naime FF, Younes RN, Kersten BG, et al. Metastatic non-small cell lung cancer in Brazil: treatment heterogeneity in routine clinical practice. Clinics. 2007;62(4):397-404. http://dx.doi.org/10.1590/S1807-59322007000400005. PMid:17823701.

16. Younes RN, Pereira JR, Fares AL, Gross JL. Chemotherapy beyond first-line in stage IV metastatic non-small cell lung cancer. Rev Assoc Med Bras. 2011;57(6):686-91. http://dx.doi.org/10.1590/S0104-42302011000600017.

Autor correspondente: 